\title{
Quantification of Acute Pulmonary Emboli using a 3D-based Computed Tomography Method: Comparison with the Qanadli score, Biomarkers, and Clinical Prognosis
}

\author{
Wei-Ming Huang \\ Mackay Memorial Hospital \\ Wen-Jui Wu \\ Mackay Memorial Hospital \\ Sheng-Hsiung Yang \\ Mackay Memorial Hospital \\ Kuo-Tzu Sung \\ Mackay Medical College
}

Ta-Chuan Hung

Mackay Junior College of Medicine, Nursing and Management

Chung-Lieh Hung ( $\nabla$ jotaro3791@gmail.com )

Mackay Medical College

Chun-Ho Yun

Mackay Memorial Hospital

\section{Research Article}

Keywords: pulmonary emboli (PE), total embolic volume (TEV), Contrast-enhanced computed tomography (CT)

Posted Date: September 9th, 2021

DOI: https://doi.org/10.21203/rs.3.rs-838785/v1

License: (c) (i) This work is licensed under a Creative Commons Attribution 4.0 International License. Read Full License 


\section{Abstract}

Background: Detailed descriptions of acute pulmonary emboli (PE) morphology, total embolic volume (TEV), and their effects upon patients' clinical presentation and prognosis remain largely unexplored.

Methods and Results: We studied 201 subjects with acute PE to the emergency department of a single medical center from April 2009 to December 2014. Patient hemodynamics, Troponin I and D-dimer levels, echocardiography, and the mortality at 30- and 90-days were obtained. Contrast-enhanced computed tomography (CT) of pulmonary structures and 3-dimensional measures of embolic burden were performed. The results showed a linear association between the greater TEV and each of the following 4 variables (increasing incidence of right ventricular (RV) dysfunction, higher systolic pulmonary artery pressure (sPAP), greater RV diameter, and RV/left ventricular $(\mathrm{LV})$ ratio (all $p<0.001)$ ). Among the measures of CT and echocardiography, TEV and RV/LV ratio were significantly associated with impending shock. In backward stepwise logistic regression, TEV, age and respiratory rate remained independent associated with impending shock (OR: $1.58,1.03,1.18$, respectively and all $p<0.005)$.

Conclusions: Total embolic burden assessed by CT-based quantification serves as a useful index for stressed cardiopulmonary circulation condition and can provide insights into RV dysfunction and the prediction of impending shock.

\section{Introduction}

Acute pulmonary embolism (PE) is a major public health concern and a potentially fatal disease. In the United States, the average incidence is about $0.1 \%$ per year, and more than 100,000 people die from acute $\mathrm{PE}$ annually ${ }^{1}$. Once a diagnosis of acute PE has been successfully established, patient risk stratification and prognostication are essential for guiding subsequent clinical management. The recent clinical guidelines published by the American Heart Association (AHA) and the European Society of Cardiology $(E S C)^{2,3}$ stratify patients based on massive, submassive, and low-risk PE categories or the Pulmonary Embolism Severity Index (PESI) ${ }^{4}$, respectively. Both incorporate key prognostic indicators such as the level of hemodynamic stability, myocardial injury, and right ventricular dysfunction.

Rapid and accurate diagnosis is critical for managing patients presenting with acute $\mathrm{PE}$, and over the last twenty years, multidetector computed tomography (MDCT) with ultra-high spatial resolution $(1-2 \mathrm{~mm})$ has become highly capable of detecting emboli within the main branches of the pulmonary arteries. This has revolutionized the diagnostic approach for acute PE and largely replaced other diagnostic tests such as ventilation-perfusion scans and invasive pulmonary angiography.

Interestingly, as the most commonly used diagnostic test for acute PE, MDCT can provide detailed information on embolic location and burden, neither of which is considered in the above guidelines to be a prognostic marker. Tuzovic et al. found that central and multilobar clots were correlated with right ventricle (RV) dysfunction ${ }^{5}$, but studies have not shown any significant correlation between clot burden, 
as measured using current two-dimensional (2D) methods, and adverse clinical outcomes ${ }^{6,7}$. In contrast, some studies have shown a correlation between a patient's clot location and burden, as measured using either 2D methods or the Mastora or Qanadli scores, and their clinical condition ${ }^{8,9}$. Praveen et al. also found that a higher clot burden was associated with right heart strain and adverse clinical events ${ }^{10}$. Previously, measuring clot burden using three-dimensional (3D) methods was impractical because it requires a large amount of manpower during daily clinical practice. Recently, however, a novel 3D-based computed tomography (3D CT) method was developed, and it has been widely applied to oncology patient images for precise tumor measurement ${ }^{11}$. As a result of the advancement of computer science and software, this 3D CT method has become more accurate and less demanding. Therefore, we aimed to assess the association between clot volume burden estimated using this novel 3D CT method and the Qanadli score, biomarkers, and clinical outcomes, including impending shock (normotension at arrival and subsequent development of hypotension requiring vasopressor) and short-term mortality.

\section{Results}

\section{Patient characteristics}

The mean age of the patient population was $66.8 \pm 17$ years, and it included 122 females $(60.7 \%)$ and 79 males (39.3\%). Their characteristics are shown in Table 1. A minority of the patients had either a history of cancer or chronic heart/lung disease (23.9\% and $45.1 \%$, respectively), and the majority $(79.3 \%)$ had a high-risk SPESI score. Echocardiographic RV dysfunction was present in $30.2 \%$ of the patients. The arrival vital signs were generally stable, with saturation $>90 \%$ (93.9 $\pm 5.6 \%$ ), systolic blood pressure $>100 \mathrm{mmHg}$ $(128.6 \pm 25.9 \mathrm{mmHg})$, and respiratory rate $<30$ per $\min (21.9 \pm 5.24$ per $\mathrm{min})$. The lab data shows that most of the patients had BNP $\leq 400(72.1 \%)$ and troponin I $\leq 0.04(55.2 \%)$ at arrival, with an average Ddimer of $10,363 \mathrm{ng} / \mathrm{mL}$. 
Table 1

Characteristics of the study population

\section{Age (yrs)}

Gender

Male

Female

History of cancer

Yes

No

History of chronic heart lung disease

Yes

No

Arrival vital signs

$\mathrm{SpO}_{2}(\%)$

SBP

DBP

RR

sPESI score

High risk $(\geq 1)$

Low risk $(<1)$

Lab data

BNP

$\mathrm{BNP}>400$

$\mathrm{BNP} \leq 400$

Troponin I

Troponin I > 0.04

Troponin I $\leq 0.04$

D-dimer
$66.8( \pm 17.0)$

79 (39.3\%)

122 (60.7\%)

$48(23.9 \%)$

153 (76.1\%)

$88(45.1 \%)$

107 (54.9\%)

$93.9( \pm 5.6)$

$128.6( \pm 25.9)$

$73.4( \pm 16.4)$

$21.9( \pm 5.24)$

142 (79.3\%)

37 (20.7\%)

$39(27.9 \%)$

101 (72.1\%)

81 (44.8\%)

100 (55.2\%)

10363 ( \pm 74701$)$

SBP: systolic blood pressure; RR: respiratory rate; ePAP: estimated pulmonary arterial pressure; RV: right ventricle; LV: left ventricle; RV/LV: ratio of diameter of RV and diameter of LV 


\begin{tabular}{|lc|}
\hline Age (yrs) & $66.8( \pm 17.0)$ \\
\hline Echocardiogram data & $42.3( \pm 16.8)$ \\
\hline ePAP & \\
\hline RV dysfunction & $42(30.2 \%)$ \\
\hline Yes & $97(69.8 \%)$ \\
\hline No & $17(8.99 \%)$ \\
\hline Mortality & $28(14.8 \%)$ \\
\hline 30 days Mortality & \\
\hline 90 days Mortality & \\
\hline $\begin{array}{l}\text { SBP: systolic blood pressure; RR: respiratory rate; ePAP: estimated pulmonary arterial pressure; RV: } \\
\text { right ventricle; LV: left ventricle; RV/LV: ratio of diameter of RV and diameter of LV }\end{array}$ \\
\hline
\end{tabular}

\section{CT findings}

The TEV was $8.5 \pm 9.2 \mathrm{~cm}^{3}$, and the embolic volume in the right and left pulmonary arterial tree was 5.26 $\pm 5.96 \mathrm{~cm}^{3}$ and $3.17 \pm 4.33 \mathrm{~cm} 3$ (Table 2). The overall Qanadli score was $6.8 \pm 4.0$. The average diameter of the RV was $4.50 \pm 0.85 \mathrm{~cm}$ and the RV/LV ratio was $1.39 \pm 0.55$. The average diameter of the pulmonary trunk was $3.01 \pm 0.49 \mathrm{~cm}$.

Table 2

CT assessments of pulmonary emboli

\begin{tabular}{|ll|}
\hline CT data & \\
\hline Total emboli volume & $8.5( \pm 9.2)\left(\mathrm{cm}^{3}\right)$ \\
\hline Qanadli score & $6.8( \pm 4.0)$ \\
\hline Left side emboli volume & $3.17( \pm 4.33)\left(\mathrm{cm}^{3}\right)$ \\
\hline Right side emboli volume & $5.26( \pm 5.96)\left(\mathrm{cm}^{3}\right)$ \\
\hline Diameter of RV & $4.50( \pm 0.85)(\mathrm{cm})$ \\
\hline RV/LV & $1.39( \pm 0.55)$ \\
\hline Diameter of pulmonary trunk & $3.01( \pm 0.49)(\mathrm{cm})$ \\
\hline
\end{tabular}

With increasing TEV quartile (Table 3), the patients exhibited poorer RV dysfunction, higher sPAP, larger RV diameter, increased RV/LV ratio, larger PT diameter, and greater incidence of abnormal troponin I levels. Age, gender, and BNP were not related to TEV in this population. In addition, $\mathrm{SpO}_{2}$ decreased significantly with increasing TEV. 
Table 3

Total emboli volume (TEV) quartiles

\begin{tabular}{|llllll|}
\hline & Q1 $(\mathbf{n = 5 0})$ & Q2 $(\mathbf{n = 5 0})$ & Q3 $(\mathbf{n = 5 0})$ & Q4 $(\mathbf{n}=\mathbf{5 1})$ & p-value \\
\hline TEV $\left(\mathrm{cm}^{3}\right)$ & 0.44 & 2.49 & 8.07 & 21.78 & \\
\hline Age & 68.06 & 68.74 & 64.40 & 66.06 & 0.337 \\
\hline Gender (male) & $40 \%$ & $34 \%$ & $40 \%$ & $43.1 \%$ & 0.820 \\
\hline RV dysfunction & $27.8 \%$ & $6.06 \%$ & $34.3 \%$ & $51.4 \%$ & $0.0007^{*}$ \\
\hline sPAP & 38.39 & 39.08 & 45.51 & 46.44 & $0.0144^{*}$ \\
\hline Diameter of RV & 4.19 & 4.20 & 4.54 & 5.05 & $<0.001^{*}$ \\
\hline RV/LV & 1.10 & 1.18 & 1.40 & 1.87 & $<0.001^{*}$ \\
\hline Diameter of PT & 2.93 & 2.97 & 2.96 & 3.16 & $0.0239^{*}$ \\
\hline SpO 2 & 96.44 & 93.03 & 93.81 & 92.30 & $0.0138^{*}$ \\
\hline BNP $>$ 400 & $25.7 \%$ & $17.6 \%$ & $24.2 \%$ & $42.1 \%$ & 0.117 \\
\hline Troponin I $>0.04$ & $29.8 \%$ & $39.5 \%$ & $41.9 \%$ & $66.7 \%$ & $0.0026^{*}$ \\
\hline PT: pulmonary trunk & & & & \\
\hline
\end{tabular}

We used multivariate logistic regression analysis to evaluate the relationship between RV dysfunction and several clinical and radiologic factors (age, gender, systolic blood pressure [SBP], respiratory rate, history of cancer, history of chronic heart/lung disease, BNP, troponin I, and TEV). The backward stepwise selection method was used for model selection. The final model included TEV and troponin I, which provided a reasonable model fit (Table 4). TEV was significantly related to the incidence of RV dysfunction $(O R=1.93, p=0.0039)$. 
Table 4

Backward stepwise logistic regression for RV dysfunction

\begin{tabular}{|lccc|}
\hline & OR & $95 \% \mathrm{Cl}$ & P-value \\
\hline Total emboli volume $\left(10 \mathrm{~cm}^{3}\right)$ & 1.93 & $1.25-3.09$ & $0.0039 *$ \\
\hline Troponin I $>0.04$ & 1.61 & $0.72-3.63$ & 0.25 \\
\hline Full model (logistic regression): & & \\
\hline $\begin{array}{l}\text { RV dysfunction } \sim \text { total emboli volume + chronic heart lung disease + age + gender + cancer + BNP + } \\
\text { Tnl + SBP + respiratory rate }\end{array}$ & \\
\hline After backward stepwise selection, the model become: & \\
\hline RV dysfunction $~$ & \\
\hline
\end{tabular}

TEV and the Qanadli score were strongly correlated ( $r=0.69, p<0.001$; Fig. 2), although the correlation was stronger when TEV was $<10 \mathrm{~cm}^{3}$ and weaker when TEV was $>10 \mathrm{~cm}^{3}$ (Fig. 2). In addition, TEV was a better predictor of RV dysfunction than the Qanadli score, according to the ROC analysis (AUC: 0.65 vs $0.58, p=0.015$; Fig. 3). Reproducibility, as evaluated using the intraclass correlation coefficient (ICC), was higher with TEV than with the Qanadli score. The ICCs of TEV and the Qanadli score were $0.99(95 \% \mathrm{Cl}$ : $0.98-0.99)$ and 0.74 (95\% Cl: 0.58-0.85), respectively.

The 30 - and 90-day mortality rates were $8.99 \%$ (17 cases) and $14.8 \%$ (28 cases), respectively, and these were not significantly correlated to TEV or the Qanadli score. The leading cause of death was cancer (Fig. 4). To evaluate the relationships between radiologic factors and impending shock, we assessed the correlation between impending shock and the echocardiogram findings (sPAP and RV dysfunction) and CT measurements (TEV, Qanadli score, RV diameter, and RV/LV ratio). TEV (OR = 1.06, 95\% Cl: 1.01-1.12, $p=0.013)$ and the $R V / L V$ ratio $(O R=2.51,95 \% \mathrm{Cl}: 1.11-5.65, p=0.02)$ were significantly correlated with impending shock (Table 6). Multivariate logistic regression analysis with backward selection was used to identify the factors related to impending shock. In addition, since the patients with cancer had poorer outcomes in our study (Fig. 4), they were excluded from further analysis of shock. The final model included TEV, age, and respiratory rate, and showed that TEV (OR: 2.20, $p=0.005)$, age (OR: 1.06, $p=$ $0.013)$, and respiratory rate $(O R=1.23, p=0.001)$ were significantly related to impending shock (Table 5$)$. 
Table 5

Backward stepwise logistic regression for impending shock

\begin{tabular}{|lccc|}
\hline & OR & $95 \% \mathrm{Cl}$ & P-value \\
\hline Total emboli volume $\left(10 \mathrm{~cm}^{3}\right)$ & 1.58 & $1.00-2.47$ & $0.044^{\star}$ \\
\hline Age & 1.03 & $1.003-1.07$ & $0.041^{*}$ \\
\hline Respiratory rate & 1.18 & $1.08-1.29$ & $0.001^{*}$ \\
\hline Full model (logistic regression): & & \\
\hline $\begin{array}{l}\text { Shock total emboli volume + chronic heart lung disease + age + gender + cancer + BNP + Tnl + SBP + } \\
\text { respiratory rate }\end{array}$ & & \\
\hline After backward stepwise selection, the model become: & & \\
\hline RV dysfunction total emboli volume + age + respiratory rate & & \\
\hline
\end{tabular}

Table 6

Different image modality for predicting impending shock

\begin{tabular}{|llll|}
\hline Factors & OR & $95 \%$ Cl & P \\
\hline Total emboli volume $\left(10 \mathrm{~cm}^{3}\right)$ & 1.06 & $1.01-1.12$ & $0.013^{*}$ \\
\hline Qanadli score & 1.09 & $0.95-1.26$ & 0.22 \\
\hline RV dysfunction (Yes vs No) & 1.25 & $0.30-4.70$ & 0.74 \\
\hline SPAP & 1.007 & $0.97-1.04$ & 0.71 \\
\hline RV/LV ratio & 2.51 & $1.11-5.65$ & $0.02^{\star}$ \\
\hline Diameter of RV & 1.76 & $0.94-3.36$ & 0.08 \\
\hline
\end{tabular}

Shock is defined as hypotension requiring vasopressor (including dopamine, norepinephrine and vasopressin).

\section{Discussion}

In this study, we described a novel 3D CT quantification method for TEV in acute PE, and we found that it was independently associated with the Qanadli score, echocardiographic findings (sPAP and RV dysfunction), $\mathrm{SpO}_{2}$, and troponin I. Most of these measurements are commonly available and can be obtained from the emergency department. Furthermore, we noticed that RV dysfunction, as assessed using echocardiography, is strongly correlated with TEV but not the Qanadli score, and that CT 3D measurements of clot burden played an important role in predicting impending shock but not short-term mortality. 
Historically, MDCT has been considered the gold standard for diagnosing patients with acute $\mathrm{PE}^{12}$. However, it has played a limited role in prognostication. In the current clinical guidelines issued by the AHA and ESC, a high RV/LV ratio is one of the CT factors associated with poor prognosis ${ }^{2,3}$. A metaanalysis showed that a RV/LV ratio $>1.0$ on CT was associated with a 2.5 -fold increased risk for all-cause mortality (OR: $2.5,95 \% \mathrm{Cl}: 1.8-3.5)^{13}$. In our study, we also found that an increased RV/LV ratio was significantly associated with an increased risk for impending shock. Conversely, TEV may be an important predictor for stratifying acute PE patients who are referred to the emergency department. Increases in SPAP were correlated with increasing TEV quartiles (Table 3) and a similar pattern was seen with RV dysfunction and troponin I, while the reverse pattern was observed with $\mathrm{SpO}_{2}$. We also found that of the MDCT and echocardiogram findings in patients without cancer, TEV and RV/LV ratio were significantly correlated with impending shock (Table 6). Furthermore, in the backward stepwise logistic regression analysis, TEV, age, and respiratory rate were independently associated with impending shock (Table 5). The presence of shock is clinically relevant to the management and prognosis of acute PE. Approximately $5 \%$ of intermediate-risk PE patients who initially show hemodynamic stability will develop hemodynamic decompensation within the first 48 hours and up to as late as five days later ${ }^{14}$.

Traditionally, for acute PE patients, the presence of RV dysfunction on echocardiography and the RV/LV ratio measured via CT are key indicators for shock. Our study suggests that precisely measured 3D embolic volume in the pulmonary arteries may be a more accurate predictor of impending shock than these two indicators. To date, there are limited data on the clinical implications of embolic burden. Herein, we have introduced the clinical feasibility of this novel imaging modality in PE patients referred to the emergency department. We propose that its use for the timely diagnosis of PE and stratification of risk for impending shock in such patients may save lives in acute settings by indicating when urgent thrombolysis or thrombectomy interventions are warranted.

For echo-based RV dysfunction (Table 3), Q2 (6.06\%) is smaller than Q1 (27.8\%). After excluding counts with only RV dilatation (diameter $<30 \mathrm{~mm}$ ), which is weak evidence of RV dysfunction, we found that the percentages of Q1 and Q2 were equal (4\%). This result supports other data (sPAP, RV diameter, RV/LV) that show that when the embolic burden is small (maximal TEV in Q2 is $<4 \mathrm{~cm}^{3}$ ), changes in cardiac morphology and function are minor. To our knowledge, no other studies prove the relationship between cardiac function and precise embolic burden, and we offer this perspective to clarify the relationship.

Compared to the Qanadli score, TEV not only more accurately assesses 3D emboli burden but is also better correlated with RV dilatation and echo-based RV dysfunction. Increasing TEV and Qanadli score can both indicate a larger clot burden in the pulmonary arterial circulation, which can lead to pressure overload and RV dilatation. In our study, TEV and the Qanadli score were well correlated, especially in the case of main pulmonary arterial involvement. However, the Qanadli score only assigns one point to each subsegmental embolus, irrespective of its length and the number of related subsegmental arteries. Therefore, this score cannot reflect the actual volume of the clot burden. Indeed, we showed that the Qanadli score is only well correlated with a smaller TEV (Fig. 2), due to this underestimation. Furthermore, as a semiquantitative method, the Qanadli score is difficult to calculate and has low reproducibility and 
high interobserver variability ${ }^{15}$. Here, we have demonstrated that TEV measurement, which is both semiautomatic and fully quantitative, has better reproducibility than the Qanadli score (ICC: 0.99 vs 0.74 , respectively).

In this study, there was no significant association between TEV and 30- and 90-day mortality rates, but this was not unexpected. There are only two small studies published before 2010 that have shown a significant association between semiquantitative clot burden assessment and short-term survival ${ }^{9,16}$, whereas several more recent studies with a large number of subjects have failed to show a significant association between the Qanadli score and short-term survival ${ }^{17,18}$. This may be due to improvements in the early diagnosis and treatment of patients with shock and cardiac arrest from acute PE, which has increased their overall survival rate. Recently, Stein et al. investigated the mortality of PE patients in the United States and showed that, from 1999 to 2017, the mortality of all high-risk patients decreased from $72.7-49.8 \%{ }^{19}$. A decreasing mortality rate for acute PE makes demonstrating a significant correlation between radiologic markers and mortality more difficult. This could explain why, despite $79.3 \%$ of our patients having a high-risk SPESI score, TEV was not significantly associated with short-term mortality. In contrast, cancer, which is a major risk factor for PE and is used in calculating the sPESI score, was the leading cause of death in our patients (Fig. 4). Similarly, other PE risk factors, such as advanced age and certain medical comorbidities ${ }^{20,21}$ can significantly affect morbidity and mortality rates, even in patients who are considered to have a low-risk PE because they are normotensive with normal biomarker levels and no RV dysfunction on imaging ${ }^{15}$. Therefore, to more comprehensively stratify the severity of acute PE and the risk of early (in-hospital or 30-day) death, additional factors such as hemodynamic status, clinical condition, RV dysfunction, and troponin I levels are important and need to be explored ${ }^{19}$.

Our study has some limitations. First, it is a retrospective study with an exclusively Asian population from a single medical center, which may represent a selection bias and limit generalizability. Second, our novel 3D CT volumetric method is only semiautomatic and still requires manual adjustments. Future studies using alternative technologies, such as artificial intelligence, for fully automatic quantification of clot burden may help to improve clinical efficiency by generating 3D volumetric results before radiologist interpretation and reducing possible manual operator errors.

In conclusion, our study demonstrated that a novel 3D CT method for quantifying acute pulmonary embolism provides results that are significantly linked to clinical condition, laboratory data, RV dysfunction, and impending shock. 3D CT measurements of clot burden may thus become a useful and feasible method for acute PE risk stratification and prognostication.

\section{Methods}

This retrospective study was approved, and all methods were performed in accordance with the relevant guidelines and regulations by the institutional review board of Mackay Memorial Hospital (no. 19MMHIS293e), Taipei, Taiwan. From April 2009 to December 2014, 1134 patients with either a clinical or a radiologic diagnosis of acute $\mathrm{PE}$ were evaluated. The medical records and images for all cases were 
reviewed by two radiologists (W.H. and C.Y.), and all images have been anonymous. A total of 201 patients were included in the final analysis after the following exclusion criteria had been applied: (1) no CT angiography for PE (i.e., they either had a CT scan that did not follow the pulmonary artery protocol or they did not have any CT imaging data); (2) no thin-slice ( $\leq 3 \mathrm{~mm}$ ) image reconstruction; (3) poor imaging quality; (4) radiologic evidence of chronic PE; (5) no significant emboli detected in the CT images; (6) thromboemboli in non-pulmonary vascular locations (e.g., venous emboli or left atrial thrombus); or (7) no echocardiography results.

\section{Clinical information}

Patient characteristics (age, gender, and history of cancer and chronic heart lung disease), their clinical presentation data (blood pressure, oxygen saturation $\left[\mathrm{SpO}_{2}\right]$, and heart rate) and cardiac biomarkers (Ddimer, B-type natriuretic peptide [BNP], and troponin I) were recorded. The simplified Pulmonary Embolism Severity Index (SPESI) ${ }^{22}$ was calculated. To evaluate the effect of embolic volume on RV function, we collected the following echocardiographic data: estimated systolic pulmonary artery pressure (SPAP); RV and left ventricle (LV) diameter; and presence of RV dysfunction. RV dysfunction was diagnosed if the echocardiogram showed any of the following morphological remodeling features: (1) morphological segmental RV abnormality (e.g., presence of akinesia of the mid-free RV wall with preserved apical contractility, as indicated by McConnell's sign) with RV diameter $\leq 30 \mathrm{~mm}$; (2) RV/LV diameter ratio > 1; or

(3) RV diameter $>30 \mathrm{~mm}^{23-25}$. To evaluate clinical prognosis, we recorded the presence of shock (defined as requiring vasopressors) at emergency-department presentation or during the course of hospitalization, 30- and 90-day mortality, and the leading cause of mortality.

\section{The CT pulmonary angiography (CTPA) protocol}

All CTPA studies were performed with a 16-slice (Somatom Sensation 16, Siemens Healthcare, Forchheim, Germany) or a 64-slice (Aquilion-64, Toshiba Medical Systems, Otawara, Japan) MDCT scanner. The standard CTPA for PE was performed according to the following scan and reconstruction protocols: scans were acquired within a single breath-hold and obtained with a detector width of $16 \times$ $0.672 \mathrm{~mm}$ or $64 \times 0.5 \mathrm{~mm}$, a tube voltage of $120 \mathrm{kVp}$, automatic exposure control (AEC) for the tube current, a $0.5 \mathrm{~s}$ gantry rotation time, and a $2 \mathrm{~mm}$ reconstructed slice thickness. The injection rate of the contrast medium, which was $80 \mathrm{~mL}$ of intravenous iohexol (Omnipaque-300) or iopromide (Ultravist-300), was $2.5-3 \mathrm{~mL} / \mathrm{s}$. Scans were performed using a bolus-tracking technique and initiated when the contrast medium was first seen in the pulmonary trunk after injection. The scan covered the region from the lung apex to the lowest hemi-diaphragm.

\section{Image analysis and 3D emboli segmentation}

All CTPA images were reviewed by a radiologist (W.H.) with eight years of experience in chest CT. Any indeterminate image interpretation was resolved after discussion and review with a senior radiologist (C.Y.) with 18 years of experience in chest CT. For each case, the PE was identified and defined as an 
intraluminal central filling defect with partial or complete occlusion of the pulmonary artery. Cases with poor imaging quality (motion artifact, high noise, or poor pulmonary artery enhancement) were excluded.

The Qanadli score ${ }^{8}$ was used for semiquantitative calculation of clot burden. Using the Qanadli score, the arterial tree of each lung was divided into 10 segmental arteries (three to the upper lobes, two to the middle and lingular lobes, and five to the lower lobes). The presence of an embolus in a segmental artery was scored 1 point and the proximal arterial level was scored points equal to the sum of the distal segmental arteries. To indicate the severity of the embolus obstruction, the following weighting factor was used: 2 for total occlusion, 1 for partial occlusion, and 0 for no thrombus observed. The maximal Qanadli score for one patient was therefore 40.

A novel 3D-based CT method was used for absolute quantitative evaluation of the total embolic volume (TEV). CTPA images were transferred to a dedicated workstation (IntelliSpace Portal [ISP] 9.0, Philips Medical Systems Nederland) and the embolic volume was measured semiautomatically. Filling defects were visually identified within the arterial tree and the contents of the filling defects were defined automatically by the workstation after several manual operation steps. The workstation also automatically interpolated the filling defect between the imaging slices and then calculated the TEV (Fig. 1). The area and volume of the filling defects were confirmed by the operator using multiplanar reformation images. Other CT parameters, such as diameter of the pulmonary trunk (PT), RV, and LV, were also measured, and the RV/LV ratio was calculated. RV and LV diameters were measured on the axial image between the inner surface of the free wall and the surface of the interventricular septum.

\section{Clinical endpoint}

To evaluate clinical prognosis, we prespecified and recorded the clinical presentation of shock (defined as requiring vasopressors) at emergency-department presentation as the primary endpoint. We further assessed 30- and 90-day all-cause mortality and rehospitalization during subsequent follow-up.

\section{Statistical analysis}

Statistical analysis was performed using R (version 3.2.3; http://www.r-project.org/). Quartiles were used to display the pattern of increasing TEV and its effects on cardiac echo, CT, Qanadli score, and lab data findings. A multivariate logistic regression model with backward stepwise selection was used to evaluate the factors affecting RV dysfunction and shock. The correlations between the different image modalities -CT (TEV, Qanadli score, RV/LV ratio, RV diameter) and cardiac echography (sPAP, presence of RV dysfunction)-and impending shock were evaluated using odds ratios (OR). Receiver operating curve (ROC) analysis was used to compare the ability of TEV and Qanadli scores to predict RV dysfunction. The threshold for statistical significance was $p<0.05$.

\section{Reproducibility}

TEV and Qanadli scores were independently measured and calculated for a random sample of 50 cases generated from the final analysis set by two experienced observers (C.Y. and W.H.) blinded to the initial 
results. The intraclass correlation coefficient was used for analysis.

\section{Declarations}

\section{Acknowledgements (not compulsory)}

None.

\section{Author contributions statement}

Wei- Ming Huang: data acquisition, conceptualization, writing

Wen-Jui Wu: clinical data acquisition, data analysis, writing

Sheng Hsiung Yang: clinical data acquisition, manuscript review

Kuo-Tzu Sung: manuscript review

Ta-Chuan Hung: manuscript review

Chung-Lieh Hung: manuscript review, data analysis, methodology, supervision

Chun-Ho Yun: manuscript review, conceptualization, methodology, supervision

\section{Additional information}

Competing interests statement: This manuscript has not been published elsewhere and is not under consideration by another journal. All authors have approved the manuscript and agree with submission to Journal of Radiological Science. There are neither conflicts of interest to declare nor funding to the authors.

Ethics approval and consent to participate: Study approval was obtained from the Mackay Memorial Hospital Institutional Review Board (IRB no. 19MMHIS293e). This 3D CT image was reconstructed from de-identified data and the informed consent was waived during institutional board review.

\section{References}

1. Apostolakis, S. \& Konstantinides, S. Acute Pulmonary Embolism. Evidence-Based Cardiology Consult, 299-309, doi:10.1007/978-1-4471-4441-0_21 (2013).

2. Jaff, M. R. et al. Management of massive and submassive pulmonary embolism, iliofemoral deep vein thrombosis, and chronic thromboembolic pulmonary hypertension: a scientific statement from the American Heart Association. Circulation 123, 1788-1830, doi:10.1161/CIR.0b013e318214914f (2011). 
3. Konstantinides, S. V. et al. 2019 ESC Guidelines for the diagnosis and management of acute pulmonary embolism developed in collaboration with the European Respiratory Society (ERS): The Task Force for the diagnosis and management of acute pulmonary embolism of the European Society of Cardiology (ESC). European heart journal 41, 543-603, doi:10.1093/eurheartj/ehz405 \%J European Heart Journal (2019).

4. Jiménez, D. et al. Prognostic models for selecting patients with acute pulmonary embolism for initial outpatient therapy. Chest 132, 24-30, doi:10.1378/chest.06-2921 (2007).

5. Tuzovic, M. et al. Regional right ventricular dysfunction in acute pulmonary embolism: relationship with clot burden and biomarker profile. The international journal of cardiovascular imaging 32, 389398, doi:10.1007/s10554-015-0780-1 (2016).

6. Bach, A. G. et al. CT pulmonary angiography findings that predict 30-day mortality in patients with acute pulmonary embolism. European journal of radiology 84, 332-337, doi:10.1016/j.ejrad.2014.11.023 (2015).

7. Subramaniam, R. M. et al. Pulmonary embolism outcome: a prospective evaluation of CT pulmonary angiographic clot burden score and ECG score. AJR. American journal of roentgenology 190, 15991604, doi:10.2214/ajr.07.2858 (2008).

8. Qanadli, S. D. et al. New CT index to quantify arterial obstruction in pulmonary embolism: comparison with angiographic index and echocardiography. AJR. American journal of roentgenology 176, 1415-1420, doi:10.2214/ajr.176.6.1761415 (2001).

9. Engelke, C., Rummeny, E. J. \& Marten, K. Acute pulmonary embolism on MDCT of the chest: prediction of cor pulmonale and short-term patient survival from morphologic embolus burden. $A J R$. American journal of roentgenology 186, 1265-1271, doi:10.2214/ajr.05.0650 (2006).

10. Hariharan, P. et al. Relation Among Clot Burden, Right-Sided Heart Strain, and Adverse Events After Acute Pulmonary Embolism. The American journal of cardiology 118, 1568-1573, doi:10.1016/j.amjcard.2016.08.025 (2016).

11. Phillip, V. et al. Influence of Sorafenib and Bevacizumab on pancreatic volume - A monocentric CT based analysis. Pancreatology: official journal of the International Association of Pancreatology (IAP) ... ] 16, 621-624, doi:10.1016/j.pan.2016.02.010 (2016).

12. Stein, P. D. et al. Multidetector computed tomography for acute pulmonary embolism. The New England journal of medicine 354, 2317-2327, doi:10.1056/NEJMoa052367 (2006).

13. Meinel, F. G. et al. Predictive Value of Computed Tomography in Acute Pulmonary Embolism: Systematic Review and Meta-analysis. The American journal of medicine 128, 747-759.e742, doi:10.1016/j.amjmed.2015.01.023 (2015).

14. Meyer, G. et al. Fibrinolysis for Patients with Intermediate-Risk Pulmonary Embolism. 370, 14021411, doi:10.1056/NEJMoa1302097 (2014).

15. Vedovati, M. C. et al. Multidetector CT scan for acute pulmonary embolism: embolic burden and clinical outcome. Chest 142, 1417-1424, doi:10.1378/chest.11-2739 (2012). 
16. Wu, A. S., Pezzullo, J. A., Cronan, J. J., Hou, D. D. \& Mayo-Smith, W. W. CT pulmonary angiography: quantification of pulmonary embolus as a predictor of patient outcome-initial experience. Radiology 230, 831-835, doi:10.1148/radiol.2303030083 (2004).

17. Furlan, A. et al. Short-term mortality in acute pulmonary embolism: clot burden and signs of right heart dysfunction at CT pulmonary angiography. Radiology 265, 283-293, doi:10.1148/radiol.12110802 (2012).

18. Nakada, K., Okada, T., Osada, H. \& Honda, N. Relation between pulmonary embolus volume quantified by multidetector computed tomography and clinical status and outcome for patients with acute pulmonary embolism. Japanese journal of radiology 28, 34-42, doi:10.1007/s11604-0090380-x (2010).

19. Stein, P. D., Matta, F., Hughes, P. G. \& Hughes, M. J. Nineteen-Year Trends in Mortality of Patients Hospitalized in the United States with High-Risk Pulmonary Embolism. The American journal of medicine, doi:10.1016/j.amjmed.2021.01.026 (2021).

20. Aujesky, D. et al. Derivation and validation of a prognostic model for pulmonary embolism. American journal of respiratory and critical care medicine 172, 1041-1046, doi:10.1164/rccm.200506-8620C (2005).

21. Wicki, J., Perrier, A., Perneger, T. V., Bounameaux, H. \& Junod, A. F. Predicting adverse outcome in patients with acute pulmonary embolism: a risk score. Thrombosis and haemostasis $84,548-$ 552(2000).

22. Jiménez, D. et al. Simplification of the pulmonary embolism severity index for prognostication in patients with acute symptomatic pulmonary embolism. Archives of internal medicine 170, 13831389, doi:10.1001/archinternmed.2010.199 (2010).

23. Gao, Y., Chen, L. \& Jia, D. A predictive tool for the assessment of right ventricular dysfunction in nonhigh-risk patients with acute pulmonary embolism. BMC pulmonary medicine 21, 42, doi:10.1186/s12890-020-01380-8 (2021).

24. Henzler, T. et al. Pulmonary embolism: CT signs and cardiac biomarkers for predicting right ventricular dysfunction. The European respiratory journal 39, 919-926, doi:10.1183/09031936.00088711 (2012).

25. Matthews, J. C. \& McLaughlin, V. Acute right ventricular failure in the setting of acute pulmonary embolism or chronic pulmonary hypertension: a detailed review of the pathophysiology, diagnosis, and management. Current cardiology reviews, 4, 49-59 https://doi.org/10.2174/157340308783565384 (2008).

\section{Figures}




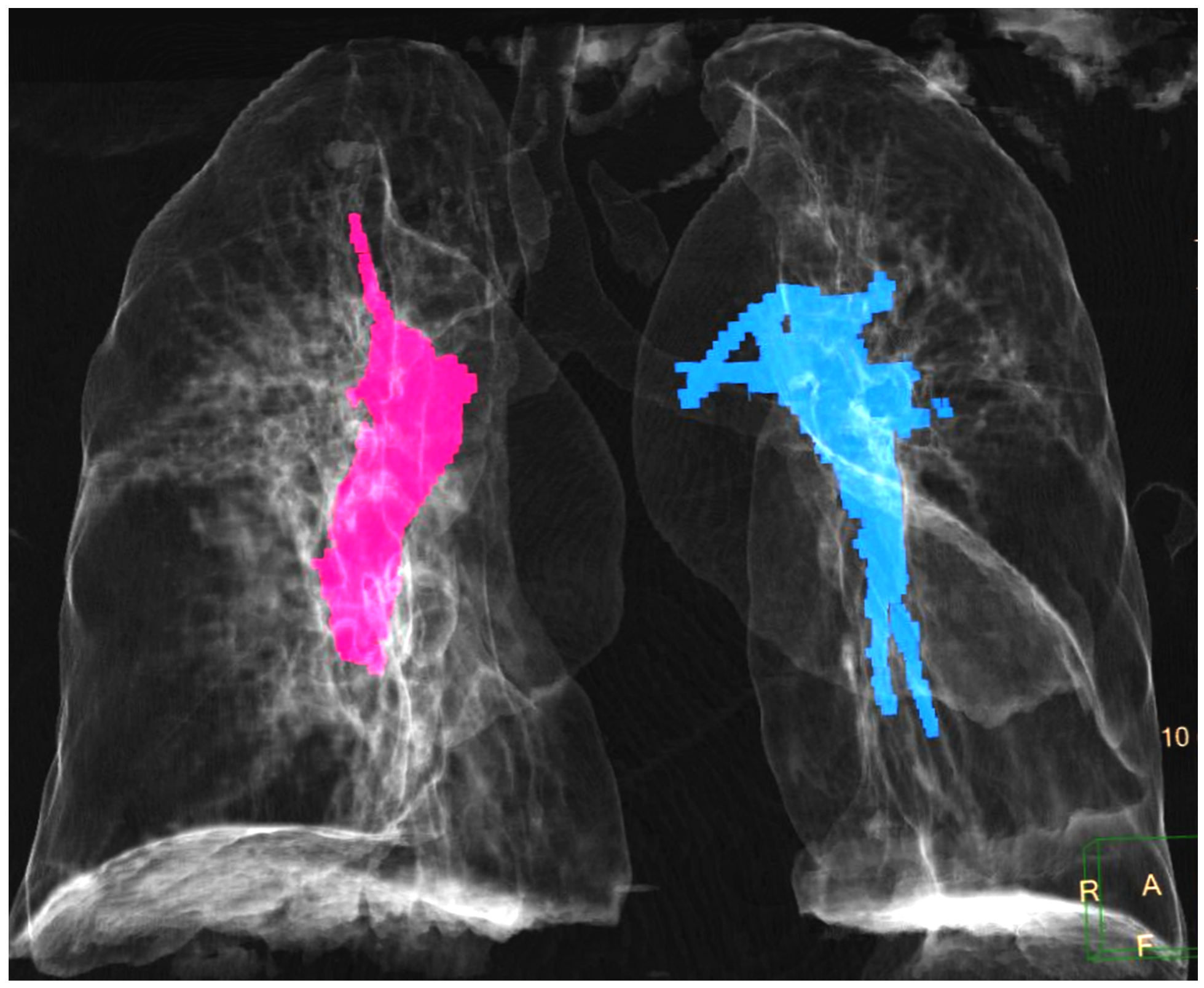

Figure 1

A 75-year-old female with high D-dimer $(7898 \mathrm{mg} / \mathrm{L})$ and mild elevated troponin-I $(0.64 \mathrm{ng} / \mathrm{mL})$. 3D CT image quantification revealed large emboli burden in the right pulmonary artery $(14.3 \mathrm{~cm} 3$, pink color) and left pulmonary artery $(15.03 \mathrm{~cm} 3$, blue color). 


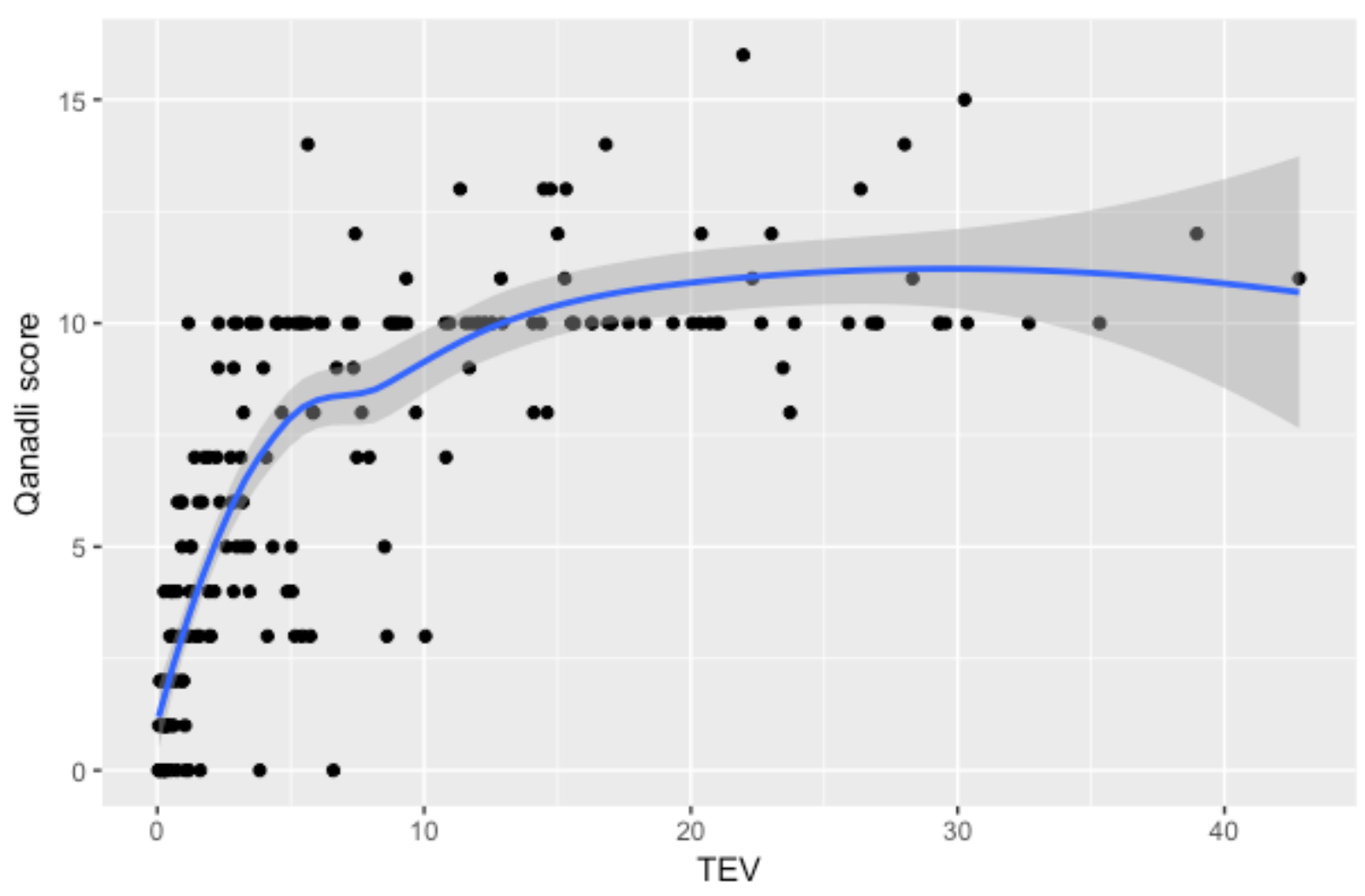

Figure 2

TEV V.S. Qanadli score The correlation of the TEV and Qanadli score $(r=0.69, p<0.001)$. However, the correlation did not seem to be linear. The regression line was drawn using the LOESS method (Local Polynomial Regression Fitting). 


\section{ROC curve}

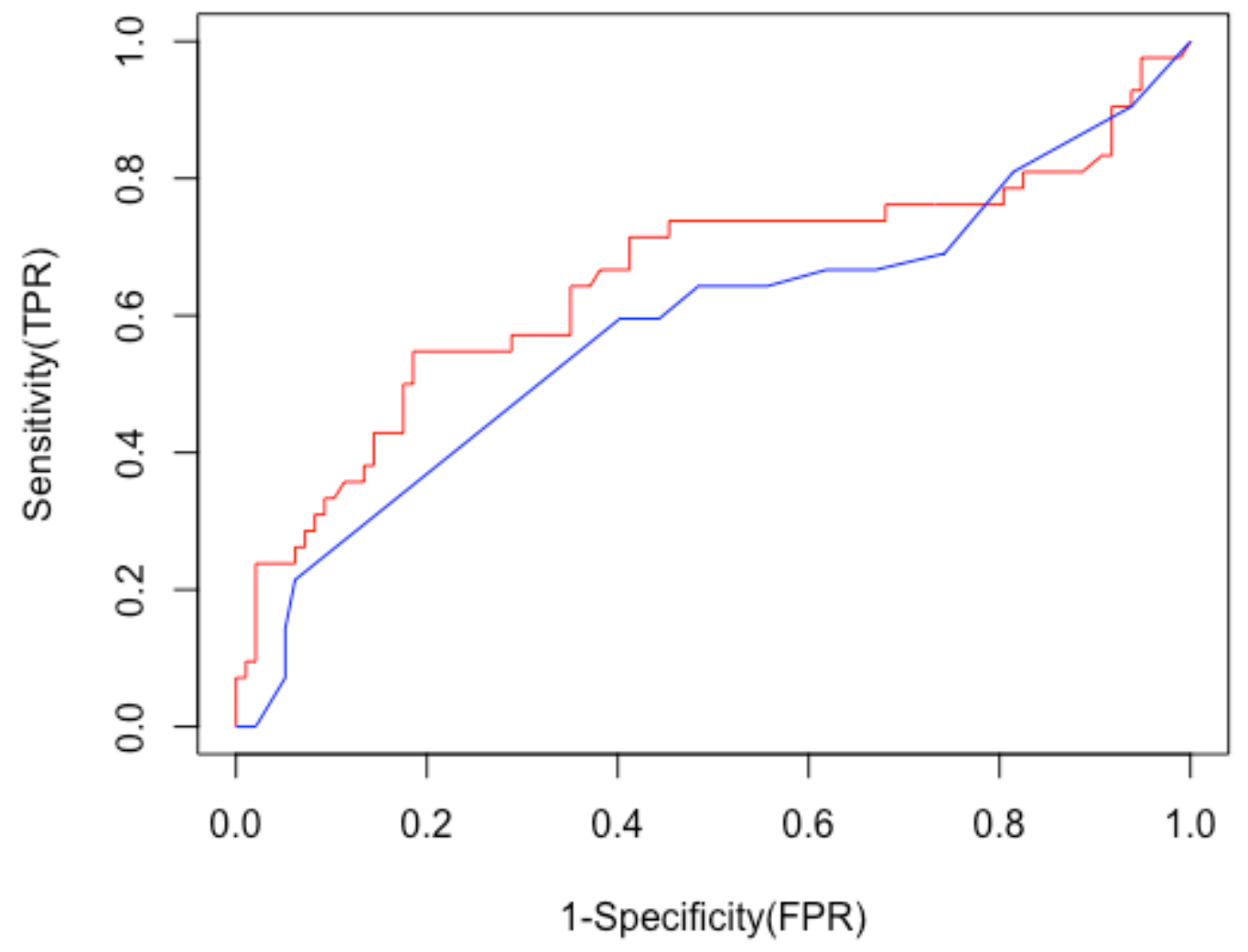

Figure 3

ROC curve to predict RV dysfunction (Red: total emboli volume, blue: Qanadli score) (AUC: 0.65 vs 0.58, p $=0.015)$ 


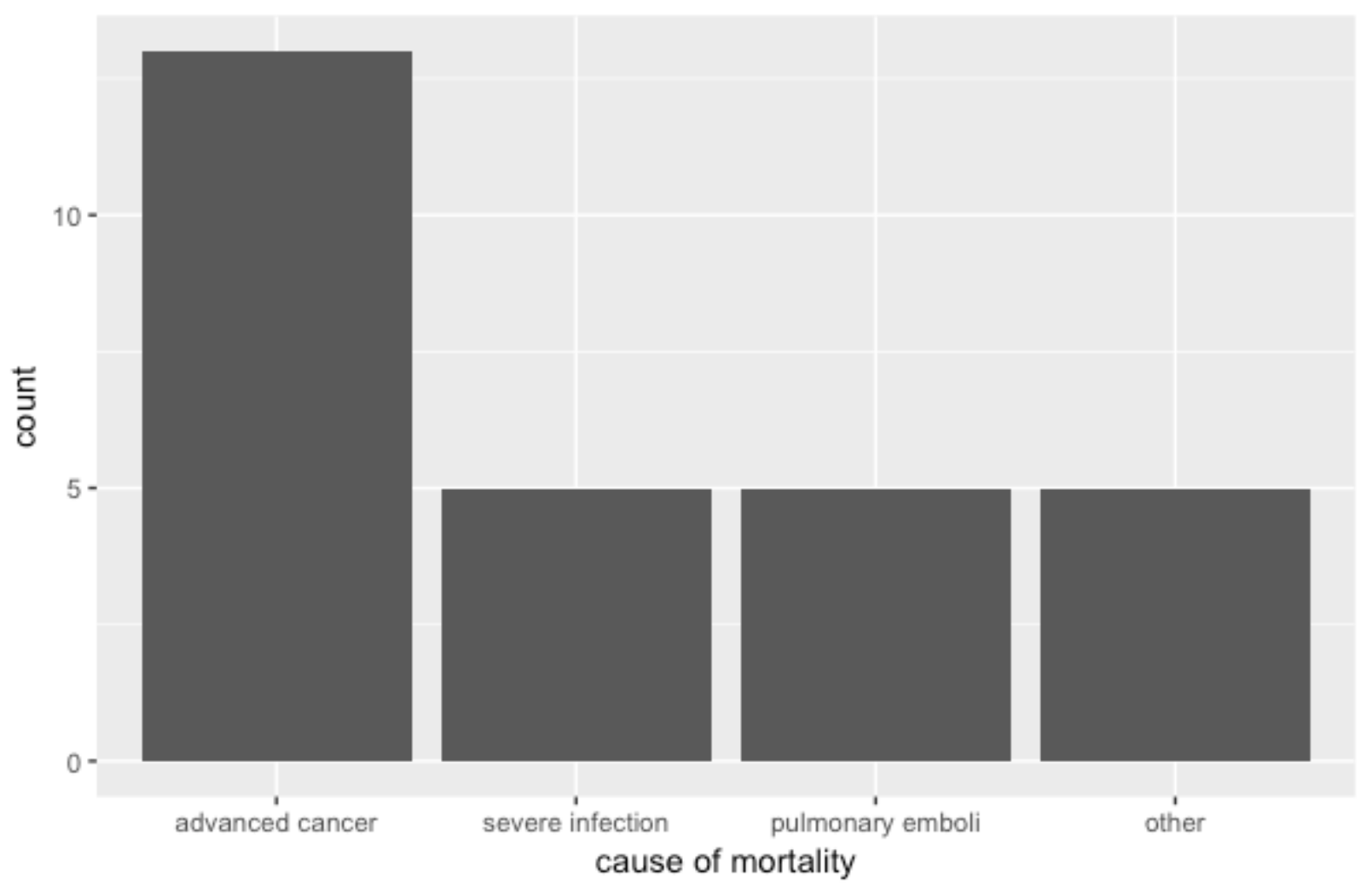

Figure 4

Cause of mortality Other: stroke, acute myocardial infarction, COPD, ESRD with hyperkalemia 